\title{
Metastatic cancer mimics interstitial lung disease. Cases when we need fast diagnosis and treatment
}

\author{
Ana Hecimovic', Marko Jakopovic ${ }^{1,2}$, Andrea Vukic Dugac ${ }^{1,2}$, Feda Dzubur ${ }^{1,2}$, Miroslav Samarzija ${ }^{1,2}$ \\ ${ }^{1}$ Department for Lung Diseases, University Hospital Centre Zagreb; ${ }^{2}$ School of Medicine, University of Zagreb, Croatia
}

\begin{abstract}
Interstitial lung diseases (ILD) are a heterogeneous group of diseases and one of the differential diagnosis which have to be excluded during diagnostic procedures are malignancies. We will present four patients who were referred to our Department because of suspicion of interstitial lung diseases according to radiology finding. In one case only, one of the radiologist's differential diagnosis was pulmonary lymphangitic carcinomatosis. All four patients had exertional dyspnea and dry cough which are nonspecific and can be first manifestation of ILD or obstructive lung diseases. After diagnostic evaluation in three cases, diagnosis was pulmonary lymphangitic carcinomatosis due to metastatic lung adenocarcinoma and in
\end{abstract}

Correspondence: Ana Hecimovic, Department for Lung Diseases, University Hospital Centre, Jordanovac 104, 10000 Zagreb, Croatia. Tel.+385.1.2385231 - Fax: +385.1.2385388.

E-mail: anahecimovic1978@gmail.com

Key words: Interstitial lung disease; neoplasm metastasis; lung neoplasms; lymphatic metastasis.

Contributions: AH, MJ, MS, conception and design of the study, manuscript drafting, critical revision; AVD, FD, data collection, critical revision of the article. All the authors read and approved the final version of the article.

Conflict of Interest: A. Hecimovic reported personal fees from Roche, Boehringer Ingelheim and MSD outside the submitted work. M. Jakopovic and M. Samarzija reported personal fees from Roche, Boehringer Ingelheim, Novartis, Astra Zeneca and Bristol-Mayers, outside the submitted work. A. Vukic Dugac reported personal fees from Astra Zeneca, Novartis, Boehringer Ingelheim and Teva, outside the submitted work. F. Dzubur reported personal fees from MSD outside the submitted work.

Funding: This research did not receive any specific grant from funding agencies in the public, commercial or not-of-profit sectors.

Received for publication: 27 January 2019.

Accepted for publication: 14 May 2019.

CCopyright A. Hecimovic et al., 2019

Licensee PAGEPress, Italy

Monaldi Archives for Chest Disease 2019; 89:1041

doi: 10.4081/monaldi.2019.1041

This article is distributed under the terms of the Creative Commons Attribution Noncommercial License (by-nc 4.0) which permits any noncommercial use, distribution, and reproduction in any medium, provided the original author(s) and source are credited. one due to metastatic adenocarcinoma of unknown primary origin. Patients with lymphangitic carcinomatosis have poor prognosis without treatment and usually die because of respiratory failure. With these four cases we want to highlight importance of thinking about malignancies when we have patients with suspicion of interstitial lung disease especially when reticular pattern is present on chest $\mathrm{X}$ ray. We also wanted to show how important is radiology finding and multidisciplinary approach, and how radiologist's differential diagnosis can be very helpful in making decisions in further investigations and way of clinicians thinking.

\section{Introduction}

Interstitial lung diseases (ILD) are a heterogeneous group of disorders and one of the differential diagnosis that we have to exclude is neoplastic process. Lung metastases are present in 30$40 \%$ of patients with malignant disease and $6-8 \%$ of this group of patients have pulmonary lymphangitic carcinomatosis (PLC) [1]. Almost any cancer has the ability to spread to the lungs and primary tumors most commonly associated with PLC are breast, stomach, lungs, pancreas and prostate [1,2]. PLC represents one of the differential diagnosis of interstitial lung abnormalities. One of the most common finding of PLC on high resolution computed tomography (HRCT) is nodular thickening of the peribronchovascular interstitium, apart from the interlobular septal thickening [3].

We will present four patients who were admitted to our Department because of radiology finding of interstitial lung changes and presumed diagnosis of ILD. After diagnostic procedures, in three cases the final diagnosis was lung cancer with PLC, and in the fourth case diagnosis was PLC from an adenocarcinoma of unknown primary origin.

\section{Cases Reports}

\section{Case \#1}

A 61-year-old non-smoker female was admitted to our Department because of progressive dyspnea and respiratory failure. She had systemic arterial hypertension in her medical history and otherwise she was healthy. She started to notice progressive dyspnea four months before admission and reported cough of 1month duration. She did not have fever and chest pain but she lost $8 \mathrm{~kg}$ in the last 3 months. She also had back pain during last 4 months and she was referred to neurologist in another hospital. MR of thoracic and lumbar spine was done which shoved bone lesions suspected of metastatic bone lesions so further investigation was suggested. Pulmonary auscultation revealed bilateral, dif- 
fuse crackles. Chest X-ray showed bilateral interstitial lesions with consolidations in the middle and lower lung fields (Figure 1). In laboratory findings there was leukocytosis (L $\left.15,6 \times 10^{9} / \mathrm{L}\right)$, elevated C-reactive protein (CRP) $93 \mathrm{mg} / \mathrm{L}$ and other findings within normal limits. Partial pressure of oxygen at admission was 34 $\mathrm{mmHg}$ and during the whole stay she was oxygen dependent on high $\mathrm{FiO}_{2}$. According to her chest X-ray, history and spine MR there was a high suspicion on metastatic malignant disease, and in spite of her sever clinical condition we decided to perform bronchoscopy with bronchoalveolar lavage (BAL). Cytology report was papillary adenocarcinoma, but further investigation was not possible due to low number of malignant cells. Further radiologic diagnostic procedures were not done due to rapid deterioration of her clinical condition. Her chest X-ray showed significant progression of bilateral opacities after only 7 days. Because of her performance status, fast clinical deterioration and the fact that we did not now the primary origin of adenocarcinoma, any oncology treatment was not possible and she died two weeks after admission.

\section{Case \#2}

The second patient was a 65-year-old male, ex-smoker with history of bilateral aortobifemoral bypass due to Leriche syndrome. He presented to our daily hospital with exertional dyspnea and dry cough for five months without fever or any pain. His laboratory findings, spirometry and arterial blood gases analysis were within normal limits but he had severely reduced diffusing capacity of lung for carbon monoxide (DLCO), 36,5\%. On chest X-ray bilateral reticular opacities were described and CT scan showed signs of advanced fibrosis in lower lobes with traction bronchiectasis and an area of ground glass opacity in left lower lobe (Figure 2 a,b). According to chest $\mathrm{CT}$ scan and low $\mathrm{CO}$ diffusing capacity first differential diagnosis was some of the fibrosing ILD like idiopathic pulmonary fibrosis or nonspecific interstitial pneumonia. Bronchoscopy with BAL from lingual and transbronchial lung biopsy (TBLB) from left lower lobe was done. BAL cytology and TBLB pathology report were lung adenocarcinoma, EGFR negative. After these results, we concluded that previously described changes on CT scan were not ILT but PLC with most probably primary tumor site in the left lower lobe. Treatment with paclitaxel with carboplatin was started but after only one cycle of therapy patient refused to continue the treatment. Five months later his condition rapidly deteriorated and he died due to respiratory failure.

\section{Case \#3}

A non-smoker, 53-year-old male with history of systemic arterial hypertension was admitted to local hospital because of progressive dyspnea, fever up to $39.7^{\circ} \mathrm{C}$ and dry cough for two months. At admission he had exertional dyspnea after only several meters of walking. Chest X-ray shoved attenuated interstitial opacities and CT scan of thorax revealed consolidations of right lung which were characterized by radiologist as chronic inflammation, right sided

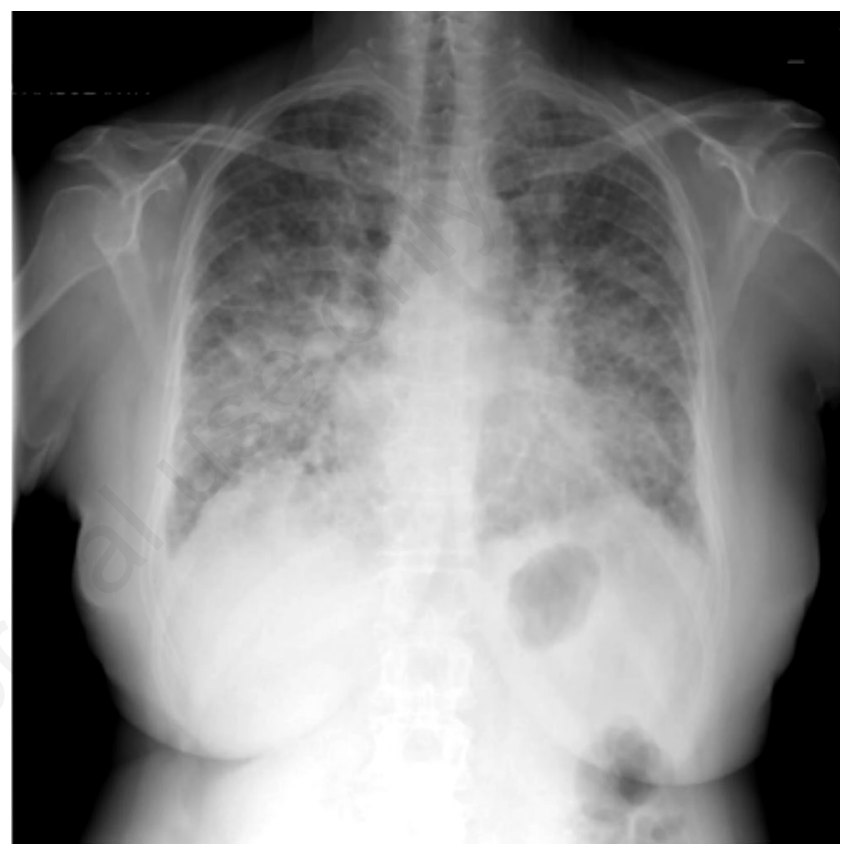

Figure 1. Case \#1. Bilateral interstitial lesions with consolidations in the middle and lower lung fields.
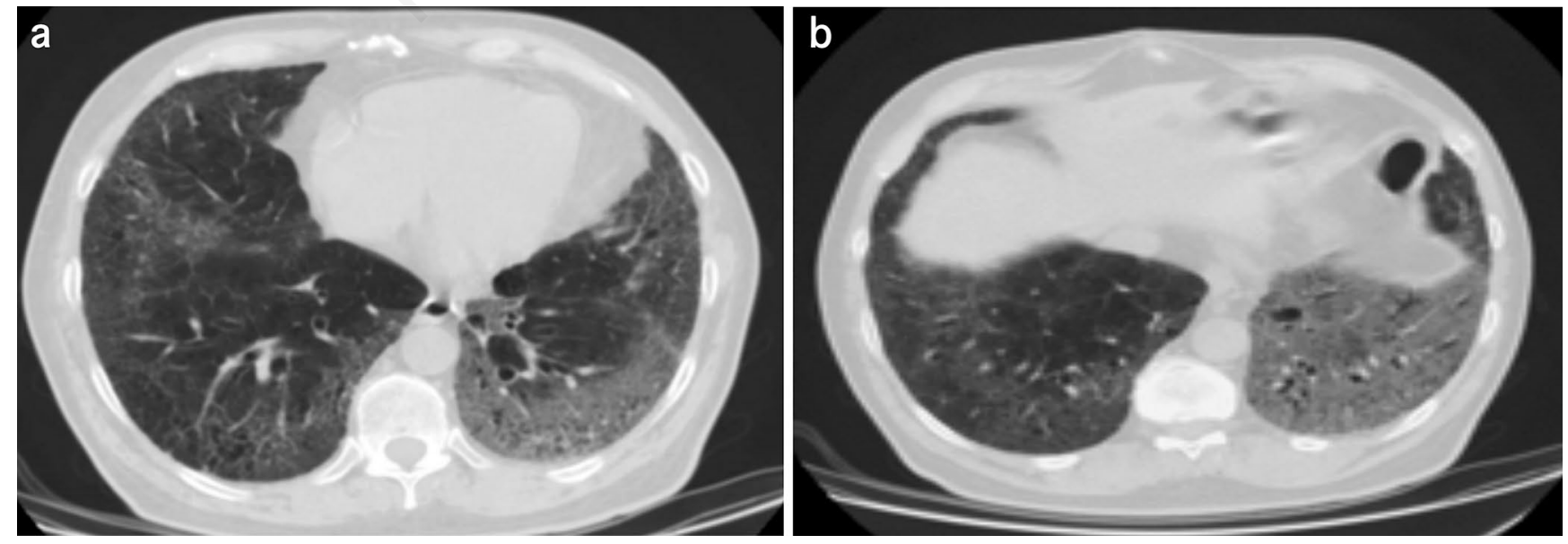

Figure 2. Case \#2. Signs of advanced fibrosis in lower lobes with traction bronchiectasis (a) and an area of ground glass opacity in left lower lobe (b). 
pleural effusion and diffuse pleural thickening. He was treated with antibiotics with no improvement on chest X-ray or in clinical condition. They did not perform further invasive diagnostic procedures due to technical reasons, and because of presumed diagnosis of ILD, he was transferred to our Department for further evaluation 5 months after his symptoms begun. At admission he had hemoptysis and severe respiratory failure with partial oxygen pressure on room air $34 \mathrm{mmHg}$. On examination he was dyspneic with bibasilar crackles on his lungs. Bilateral alveolar and interstitial opacities were in progression on chest X-ray with elevated total leucocyte count 29.9 $\mathrm{x} 10^{9} / \mathrm{L}$ and CRP $110.9 \mathrm{mg} / \mathrm{L}$. Chest CT scan this time revealed diffuse ground-glass opacities with consolidations, interlobular and intralobular septal and interstitial thickening, right- sided pleural effusion and pleural masses (Figure 3). Bronchoscopy was performed with TBLB and BAL, and both cytology and pathology finding was consistent with lung adenocarcinoma. Consolidation of the right side of lungs was primary adenocarcinoma and other changes revealed on CT scan were due to PLC and pleural carcinosis. We started treatment with paclitaxel and carboplatin which was standard of care according to our insurance company at that time. After first two cycles his clinical condition was slightly improved but after 3 cycles his condition severely deteriorated and he died four months after diagnosis was established.

\section{Case \#4}

A 79-year-old male, ex-smoker (history of 25 pack-year) was transferred to our Department from another hospital because of suspicion of ILD. He reported diabetes mellitus types 2 for the last 15 years and prostatic operation due to hypertrophy in his medical history. His symptoms started 4 weeks before admission with dry cough and shortness of breath without fever or chest pain. Chest X-ray showed bilateral interstitial infiltrates and he was treated with antibiotics with no improvement. At admission he was oxygen-dependent, flow rate of $2 \mathrm{~L} / \mathrm{min}$ via nasal cannula. His laboratory findings revealed anemia $(\mathrm{Hb} 121 \mathrm{~g} / \mathrm{L})$ and slightly elevated CRP $23 \mathrm{mg} / \mathrm{L}$. CT scan of thorax showed nodular interstitial lesions, discrete interlobar septal thickening, bilateral hilar and mediastinal lymphadenopathy and pleural effusion on the right side (Figure 4). His spirometry showed restrictive pattern and he was not able to perform DLCO due to cough. Thoracentesis was done and cytology analysis accompanied with immunocytochemistry of exfoliated cells revealed metastatic lung adenocarcinoma (cells were CK 7 and TF1 positive and CK20 negative), EGFR negative. Due to finding of chest CT scan and cytology results definitive diagnosis was lung adenocarcinoma with PLC and pleural carcinosis. Treatment with chemotherapy was started, he received two cycles of pemetrexed with carboplatinum, but thereafter his condition deteriorated and further treatment was not indicated due to his performance status. The patient died 6 months after first admission to the hospital.

\section{Discussion}

Lung is a common site of metastases, and typical radiologic presentation of pulmonary metastases are multiple peripherally located round variable-sized nodules in a case of hematogenous metastasis and in the case of PLC diffuse thickening of interstitium [4]. The PLC is characterized by diffuse infiltration and obstruction of the pulmonary lymphatic system by tumor cells [1,2]. There are two ways of spreading tumor cells through lymphatics: the first is resulting from the hematologic dissemination of the primary neoplasia and extending from the capillaries to the lymphatic system, and
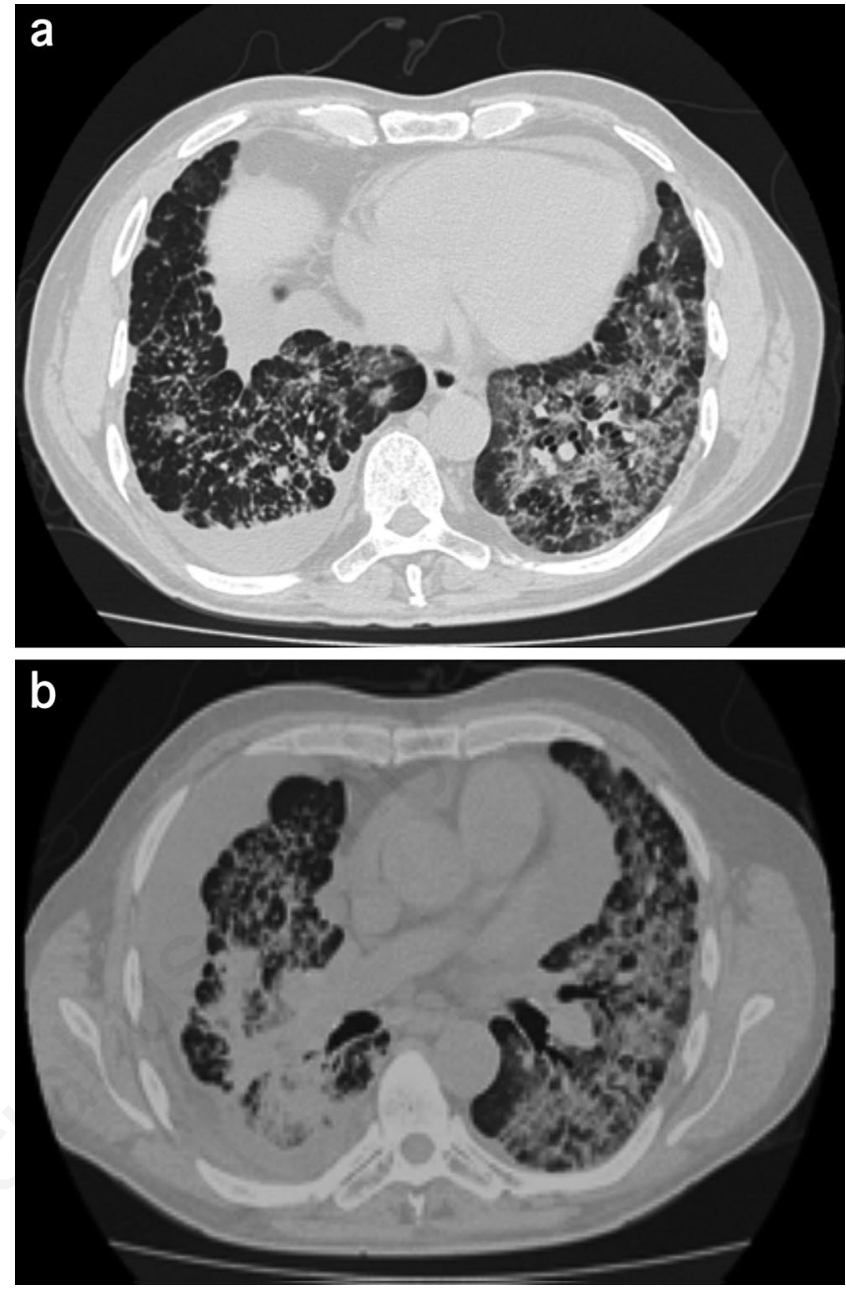

Figure 3. Case \#3. Interlobular and intralobular septal and interstitial thickening, pleural effusion on the right side (a) and right sided pleural thickening (b).

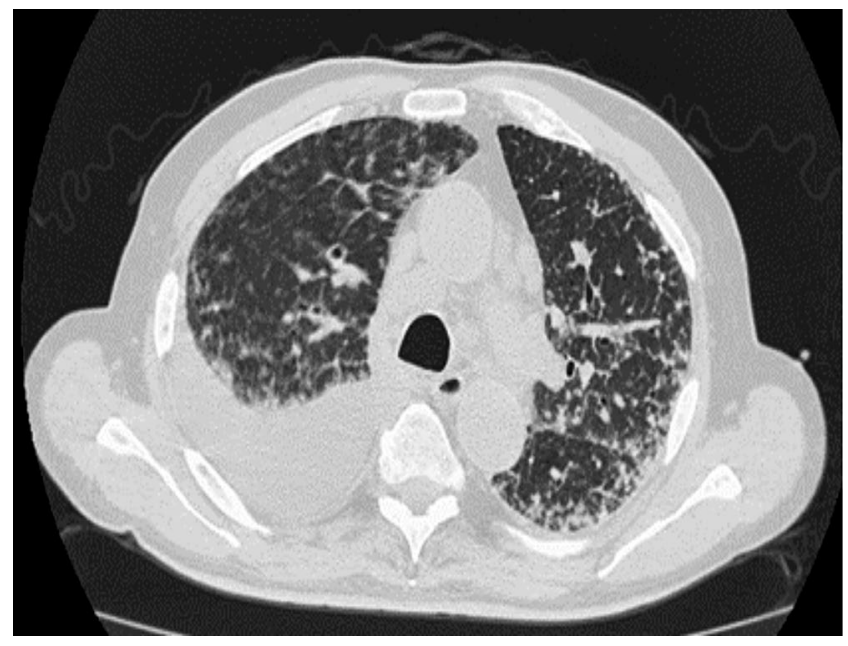

Figure 4. Case \#4. Interstitial lesions, discrete interlobar septal thickening, and pleural effusion on the right side. 
the second is the retrograde dissemination from mediastinal and hilar lymph nodes toward parenchyma [5]. Any metastatic neoplasm can cause PLC [6] but $80 \%$ of PLC are caused by metastatic adenocarcinoma [7]. Lungs are primary site of the PLC in $17 \%$ of cases $[1,2]$.

Lung biopsy (transbronchial or open) is preferred diagnostic procedure for PLC but Masson et al showed that pulmonary microvascular cytology may be of value when lung biopsy is refused or thought to be too hazardous [8].

We presented four patients whose first presentation of the disease were respiratory symptoms due to PLC and in two cases it was followed by severe respiratory failure. In cases of PLC symptoms of primary cancer are usually absent or masked by pulmonary symptoms more characteristic for ILD and the frequency of this presentation is unknown. Typical clinical presentation are progressive dyspnea and dry cough which we saw in all our cases. From our experience duration of symptoms is usually from few weeks to few months with rapid progression. Cough is first presentation in about sixty percent of patients and occasionally accompanied by sputum which is ranged from white to blood tinged [7].

Chest radiographic features of PLC are coarse bronchovascular markings with irregular outline, coarse reticulonodular pattern with intraparenchymal extension of tumor, bilateral changes predominately in the lower lobes of the lungs (although changes may be unilateral or asymmetric) and Karley A and B lines [2].

In $30 \%$ to $50 \%$ of patients with PLC pleural effusions are present, and in $20 \%$ to $40 \%$ cases hilar or mediastinal lymphadenopathy can be revealed [7]. In a case of ILD pleural effusions are less frequent and it can be found according to Highland and Heffner in 6\% of patients with idiopathic pulmonary fibrosis, in sarcoidosis effusions are usually small and rare likewise in rheumatoid arthritis [9]. Pleural effusions accompanied with interstitial lung disease most frequently occurs in systemic lupus erythematosus and it could be found in up to $50 \%$ of cases [9]. Pleural effusions can be drug induced and we can find interstitial lung changes and effusions at the same time. When we have radiologic finding of interstitial lung changes and pleural effusion, without clinical or laboratory findings of connective tissue disease, it is important to exclude some other diagnosis like malignancies or adverse effects of treatment.

Computed tomography findings of PLC can reveal smooth (early stage) and nodular (late stage) thickening of interlobular septa and peribronchovascular interstitium, polygonal arcades with thickened limbs from thickened septa of adjacent lobules, maintained normal lung architecture, ground-glass appearance from interstitial edema or extension of the tumor into parenchyma $[2,10]$.

PLC can mimic sarcoidosis because nodular thickening and ground-glass are seen in 30\%-60\% patients with sarcoidosis [2]. Sarcoidosis is characterized by middle and upper zone predominance while in PLC changes are usually found in lower lobes. For distinguishing PLC from sarcoidosis preservation of the architecture of the secondary pulmonary lobule and the less common incidence of mediastinal lymphadenopathy can help us [11]. In our fourth case, one of the differential diagnosis according to radiologist's finding was sarcoidosis but to confirm the diagnosis biopsy is necessary.

When we have patients with rapid onset and progressive symptoms, predominant changes in lower lobes, pleural effusion accompanied to interstitial changes, asymmetrically enlarged lymph nodes and no response to corticosteroids treatment we have to exclude some other diagnosis, like malignancies, rather than sarcoidosis.

In our second case one of the differential diagnosis was idiopathic pulmonary fibrosis because we had thickening of the interlobular septa without nodular pattern which can be also found as a presentation of PLC or pulmonary edema. Only in the first case radiologist suggested PLC as differential diagnosis, and in all other three cases some of the ILDs were suspected according to radiologist's findings. This can be crucial because with most of the ILDs we have better prognosis and more time for diagnostic procedures, and when we have suspicion of PLC we have to act fast. By these cases we also wanted to point out how multidisciplinary approach is very important and experienced radiologist and their findings can be of enormous help to clinicians.

Patients with PLC have poor prognosis and without treatment less than $50 \%$ of patients who present with respiratory symptoms survive 3 months [1]. PLC may cause significant deterioration of patient's condition and most of the patients die as a result of respiratory failure. Early diagnosis is important and crucial for improving the survival of patients with PLC. In our cases we showed that patient's condition can deteriorate in only few weeks and if diagnose is established too late, survival is only few months despite the treatment.

\section{Conclusions}

Clinicians have to think about PLC when they have patients with hypoxemia and presumed ILD based on chest X-ray, especially when pleural effusion is also present. Radiologist are very important with their differential diagnosis which can be crucial for further diagnostic procedures. All our patients deteriorated in very short period of time, so due to poor prognosis and rapid clinical deterioration it is of great importance to establish diagnosis and to start treating these patients as soon as possible.

\section{References}

1. Bruce DM, Heys SD, Eremin O. Lymphangitis carcinomatosa: a literature review. J R Coll Surg Edinb 1996;41:7-13.

2. Thomas A, Lenox R. Pulmonary lymphangitic carcinomatosis as a primary manifestation of colon cancer in a young adult. CMAJ Can Med Assoc J 2008;179:338-40.

3. Oikonomou A, Prassopoulos P. Mimics in chest disease: interstitial opacities. Insights Imaging 2013;4:9-27.

4. Seo JB, Im J-G, Goo JM et al. Atypical pulmonary metastases: Spectrum of radiologic findings. RadioGraphics 2001;21:403-17.

5. Martynychen MG, Rabelo LM, Silva RLF, et al. Carcinomatous lymphangitis as the initial manifestation of ovarian adenocarcinoma. J Bras Pneumol 2007;33:609-11.

6. Moubax K, Wuyts W, Vandecaveye V, et al. Pulmonary lymphangitic carcinomatosis as a primary manifestation of gastric carcinoma in a young adult: a case report and review of the literature. BMC Res Notes 2012;5:638.

7. Khachekian A, Shargh S, Arabian S. Pulmonary lymphangitic carcinomatosis from metastatic gastric adenocarcinoma: case report. J Am Osteopath Assoc 2015;115:332-7.

8. Masson RG, Krikorian J, Lukl P, et al. Pulmonary microvascular cytology in the diagnosis of lymphangitic carcinomatosis. N Engl J Med 1989;321:71-6.

9. Highland KB, Heffner JE. Pleural effusion in interstitial lung disease. Curr Opin Pulm Med 2004;10:390-6.

10. Davis SD. CT evaluation for pulmonary metastases in patients with extrathoracic malignancy. Radiology 1991;180:1-12.

11. Johkoh T, Ikezoe J, Tomiyama N, et al. CT findings in lymphangitic carcinomatosis of the lung: correlation with histologic findings and pulmonary function tests. AJR Am J Roentgenol 1992;158:1217-22. 